\title{
Existence of Positive Radial Solutions for a Nonvariational Polyharmonic System
}

Yajing Zhang and Jianghao Hao ${ }^{1}$

\begin{abstract}
In this work we first prove a new Liouville-type result for a polyharmonic system in $\mathbb{R}^{N}$, then prove the existence of positive radial solutions for a polyharmonic system without variational structure in bounded domains via topological method.

Mathematics Subject Classification (2000). Primary 35J60; Secondary 35J55.

Keywords. Polyharmonic system, positive radial solution, a priori estimate, existence.
\end{abstract}

\section{Introduction}

In this paper we study the existence of positive radial solutions of the following polyharmonic system

$$
\left\{\begin{array}{l}
(-\Delta)^{m} u=u^{k} v^{p}, \quad \text { in } B_{R}, \\
(-\Delta)^{m} v=u^{q} v^{s}, \quad \text { in } B_{R}, \\
u=(-\Delta) u=\cdots=(-\Delta)^{m-1} u=0, \quad \text { on } \partial B_{R}, \\
v=(-\Delta) v=\cdots=(-\Delta)^{m-1} v=0, \quad \text { on } \partial B_{R},
\end{array}\right.
$$

where $m \geq 1, N>2 m, p, q, k, s \geq 0$ and $B_{R}$ is the ball centered at zero and radius $R$ in $\mathbb{R}^{N}$.

For $m=1$, this type problem were well studied, more precisely, the existence of positive solutions of the elliptic system

$$
\left\{\begin{array}{l}
-\Delta u=f(u, v), \quad \text { in } \Omega, \\
-\Delta v=g(u, v), \quad \text { in } \Omega,
\end{array}\right.
$$

The first author was supported by NSFC (Grant No. 10701051) and China Postdoctoral Science Foundation.

The second author was supported by NSFC (Grant No. 10701051).

${ }^{1}$ Corresponding author. 
subject to zero Dirichlet boundary conditions has been studied by a number of authors, see for examples $[2-5,8,12,13,17]$, and the references therein. Variational methods are of some use when the differential problem(equations or systems) is the Euler-Lagrange equation of some functional. In the case of a scalar equation, this is always the case, provided some growth conditions on nonlinearity is imposed. For the case of a system like (1.2), it is not always the case that there is a natural functional associated to it. If the system is not variational one has to use topological methods.

Polyharmonic equations (systems) have received great attention recently, Bartsch and Guo [1] investigated semilinear elliptic systems for the polyharmonic operator having a critical growth nonlinearity. They establish conditions for existence of nontrivial solutions to the systems using variational methods. System (1.1) need not have a variational structure, and consequently we will use topological methods to prove the existence of positive solutions. The purpose of this paper partly is to show that how the topological method works in the study of higher order elliptic boundary value problems. Our main result is the following.

Theorem 1.1. Suppose that $N>2 m$, and $p, q, k, s$ satisfy

(i) $p, q>0, p q>1,0 \leq k \leq 1,0 \leq s \leq 1$,

(ii) $\frac{N}{2 m}<\min \left\{\frac{(p+1)(q+1)-k s}{p q-(1-k)(1-s)}, \frac{(p+1)(q+1)+k(q+1)+s(p+1)}{p q-1+k(q+1)+s(p+1)}\right\}$.

Then (1.1) possesses a positive radial solution.

The proof of Theorem 1.1 is based on a fixed point theorem on positive cones as well as a priori estimates for the solutions. One important feature of the proof of Theorem 1.1 is that it applies to general nonlinearities with certain growth restriction in $u$ and $v$ at infinity. Here the a priori bounds for the solutions of (1.1) are obtained via the so-called blow-up method intruduced by Gidas and Spruck in their fundamental paper [7]. Roughly speaking, in this method one assumes by contradiction that no universal estimate for the solutions. Then after some limit procedures one ends up with a limiting problem for which there are positive solutions. A contradiction comes if we prove the nonexistence of positive solutions of the limiting problem (Liouville-type theorems).

To perform our program we first need to study nonexistence of positive radial solutions for the following polyharmonic system

$$
\left\{\begin{aligned}
(-\Delta)^{m} u & =u^{k} v^{p}, \\
(-\Delta)^{m} v & =u^{q} v^{s},
\end{aligned} \quad \text { in } \quad \mathbb{R}^{N} .\right.
$$

Compared with the case $m=1$, the main difficulty we meet is that the maximum principle can not be directly applied to $u, v$ if one does not know enough information about $(-\Delta)^{i} u,(-\Delta)^{i} v, i=1, \ldots, m-1$. Hence, we need to prove the general superharmonic property of the solutions of the polyharmonic system (1.3). This is the key part of proof of the nonexistence of positive solutions. Let $(u, v) \in C^{2 m}\left(\mathbb{R}^{N}\right) \times C^{2 m}\left(\mathbb{R}^{N}\right)$ be a positive solution of the system (1.3), we say that $(u, v)$ satisfies the general superharmonic property if $(-\Delta)^{i} u \geq 0$, 
$(-\Delta)^{i} v \geq 0, i=1, \ldots, m-1$. We obtain a new Liouville-type theorem which extends the earlier works by the first author and his co-workers [10,15] to more complicated nonlinearity including the product of two powers.

The organization of the paper is as follows. In Section 2 we prove a new nonexistence result for polyharmonic system in $\mathbb{R}^{N}$. In Section 3 we prove the a priori estimates of positive solutions for the poyharmonic systems (1.1) via the blow-up method. Finally in Section 4, we use a fixed point theorem to prove Theorem 1.1. Throughout this paper, the constant $c$ will denote various generic constants.

\section{Liouville-type theorem}

In this section, we prove a Liouville type result for polyharmonic system.

Theorem 2.1. Suppose that $N>2 m$, and $p, q, k, s$ satisfy

(i) $p, q>0, p q>1,0 \leq k \leq 1,0 \leq s \leq 1$,

(ii) $\frac{N}{2 m}<\min \left\{\frac{(p+1)(q+1)-k s}{p q-(1-k)(1-s)}, \frac{(p+1)(q+1)+k(q+1)+s(p+1)}{p q-1+k(q+1)+s(p+1)}\right\}$.

Then the polyharmonic system

$$
\left\{\begin{array}{l}
(-\Delta)^{m} u=u^{k} v^{p}, \quad \text { in } \quad \mathbb{R}^{N}, \\
(-\Delta)^{m} v=u^{q} v^{s},
\end{array}\right.
$$

has no positive radial classical solutions.

We note that the above nonexistence result can be extended to more complicated system

$$
\left\{\begin{aligned}
(-\Delta)^{m} u & =|x|^{\alpha} u^{k} v^{p}, \\
(-\Delta)^{m} v & =|x|^{\beta} u^{q} v^{s},
\end{aligned} \quad \text { in } \quad \mathbb{R}^{N},\right.
$$

where $\alpha, \beta, k, s, p$ and $q \geq 0$. The proof is along the lines of Theorem 2.1.

The following lemma is a general version of Lemma 3.1 in [14].

Lemma 2.2. Suppose $y=y(r) \geq 0$ satisfies

$$
y^{\prime \prime}(r)+\frac{N-1}{r} y^{\prime}(r)+\varphi(r) \leq 0, \quad r>r_{0} \geq 0,
$$

with $\varphi$ non-negative and non-increasing, and $y^{\prime}\left(r_{0}\right) \leq 0$. Then

$$
y(r) \geq c r^{2} \varphi(r), \quad \text { for } \quad r \geq 2 r_{0},
$$

where $c=c(N)$.

Proof. From (2.2),

$$
y^{\prime \prime}(r)+\frac{N-1}{r} y^{\prime}(r) \leq 0,
$$

multiplying the above inequality by $r^{N-1}$ and integrating, we get

$$
y^{\prime}(r) \leq 0, \quad \text { for } \quad r \geq r_{0} .
$$


Multiplying (2.2) by $r^{N-1}$ and integrating the inequality, we get, for $r>2 r_{0}$,

$$
\begin{aligned}
-y^{\prime}(r) & \geq r^{1-N} \int_{r_{0}}^{r} t^{N-1} \varphi(t) d t \\
& \geq r^{1-N} \int_{r_{0}}^{r / 2} t^{N-1} \varphi(t) d t \\
& \geq \frac{1}{N} \varphi\left(\frac{r}{2}\right)\left(\frac{r}{2^{N}}-\left(\frac{r_{0}}{r}\right)^{N-1} r_{0}\right) \\
& \geq \frac{1}{N} \varphi\left(\frac{r}{2}\right)\left(\frac{r}{2^{N}}-\frac{1}{2^{N-1}} r_{0}\right) .
\end{aligned}
$$

Integrating this from $r$ to $2 r$ then yields

$$
\begin{aligned}
y(r) & \geq y(2 r)+\frac{1}{N} \int_{r}^{2 r} \varphi\left(\frac{t}{2}\right)\left(\frac{1}{2^{N}} t-\frac{1}{2^{N-1}} r_{0}\right) d t \\
& \geq \frac{1}{N} \varphi(r)\left(\frac{1}{2^{N}} 3 r^{2}-\frac{1}{2^{N-1}} r_{0} r\right) \\
& \geq \frac{1}{2^{N} N} \varphi(r) r^{2} .
\end{aligned}
$$

The proof of Lemma 2.2 is completed.

Lemma 2.3. Suppose positive radial functions $u, v$ satisfy

$$
\begin{cases}(-\Delta)^{m} u(r) \geq v^{p}(r), & r \geq r_{0}, \\ (-\Delta)^{m} v(r) \geq u^{q}(r), & r \geq r_{0}\end{cases}
$$

where $p, q>0, p q>1$ and $r_{0} \geq 0$. Then

$$
(-\Delta)^{i} u(r) \geq 0, \quad(-\Delta)^{i} v(r) \geq 0, \quad \text { for } \quad r \geq r_{0}, \quad i=1, \ldots, m-1 .
$$

The proof of this lemma can be found in [10] and [16].

Remark 2.4. It is easy to see that we can obtain the same result for the following polyharmonic inequality $(-\Delta)^{m} u \geq u^{p}, r \geq r_{0}$, where $p>1$.

Lemma 2.5. If $\psi \in C^{2 m}\left(\mathbb{R}^{N}\right)$ is positive, radial and $(-\Delta)^{k} \psi \geq 0$ in $\mathbb{R}^{N}, k=$ $1, \ldots, m$, then for every $r \in(0,+\infty)$ we have

$$
r \psi^{\prime}(r)+(N-2 m) \psi(r) \geq 0 .
$$

For its proof, see [3].

Remark 2.6. Note that $(2.4)$ is equivalent to $\left(r^{N-2 m} \psi(r)\right)^{\prime} \geq 0$. Thus $r^{N-2 m} \psi(r)$ is non-decreasing.

Proposition 2.7. If $(u, v)$ is a positive radial solution of the polyharmonic system

$$
\left\{\begin{aligned}
(-\Delta)^{m} u & =a(|x|) u^{k} v^{p}, \quad \text { in } \quad \mathbb{R}^{N}, \\
(-\Delta)^{m} v & =b(|x|) u^{q} v^{s},
\end{aligned}\right.
$$

and $a, b, p, q, k, s$ satisfy 
(i) $a, b \in C[0,+\infty)$ are non-decreasing and $a(r)>0, b(r)>0$ for $r>0$,

(ii) $0 \leq k, s \leq 1, p>0, q>0, p q>1$.

Then

$$
(-\Delta)^{i} u \geq 0, \quad(-\Delta)^{i} v \geq 0, \quad i=1, \ldots, m-1 .
$$

Proof. Step 1. We show that $(-\Delta)^{m-1} u \geq 0,(-\Delta)^{m-1} v \geq 0$.

The proof is by contradiction argument. Suppose the contrary, then there exists a number $r^{*} \geq 0$ such that $(-\Delta)^{m-1} u\left(r^{*}\right)<0$. Set $w=(-\Delta)^{m-1} u, z=$ $(-\Delta)^{m-1} v$. Then it is easy to see $-\Delta w=(-\Delta)^{m} u \geq 0$. Multiply the inequality by $r^{N-1}$ and integrate the resulting inequality, we have $\frac{d w}{d r} \leq 0$, thus $w$ is nonincreasing and

$$
w(r) \leq w\left(r^{*}\right)<0, \quad \text { for } \quad r \geq r^{*} .
$$

In the following, we discuss two cases.

Case 1. $m$ is an odd number.

It follows from (2.6),

$$
\Delta^{m-1} u \leq w\left(r^{*}\right)
$$

Multiplying the above inequality by $r^{N-1}$ and integrating twice, we get there exists $c_{1}>0$ and $r_{1}>0$ such that

$$
\Delta^{m-2} u(r) \leq c_{1} w\left(r^{*}\right) r^{2}, \quad \text { for } \quad r \geq r_{1} .
$$

Repeat the above process step by step, we get there exists $c_{2}>0$ and $r_{2}>0$ such that

Since $w\left(r^{*}\right)<0$, we have

$$
u(r) \leq c_{2} w\left(r^{*}\right) r^{2(m-1)}, \quad \text { for } \quad r \geq r_{2} .
$$

$$
u(r) \rightarrow-\infty, \quad \text { as } \quad r \rightarrow+\infty .
$$

This is a contradiction since $u(r)$ is positive.

Case 2. $m$ is an even number.

It follows from (2.6),

$$
\Delta^{m-1} u \geq-w\left(r^{*}\right)>0 .
$$

By the same arguments as we did in the proof of Case 1, we get

$$
u(r) \rightarrow+\infty, \quad \text { as } r \rightarrow+\infty .
$$

In the following, we show $(-\Delta)^{i} v \geq 0, i=1, \ldots, m-1$. If there exists $r_{3} \geq 0$ such that $z\left(r_{3}\right)=(-\Delta)^{m-1} v\left(r_{3}\right)<0$, we have

$$
\Delta^{m-1} v(r) \geq-z\left(r_{3}\right)>0, \quad \text { for } \quad r \geq r_{3},
$$

since $z$ is non-increasing and $m$ is an even number. Thus

$$
v(r) \rightarrow+\infty, \quad \text { as } \quad r \rightarrow+\infty .
$$

Since $a, b$ are non-decreasing, by (2.5), (2.9), (2.10) there exists $c_{0}>0, r_{0}>0$ such that

$$
\begin{cases}\Delta^{m} u \geq c_{0} v^{p}, & r \geq r_{0}, \\ \Delta^{m} v \geq c_{0} u^{q}, & r \geq r_{0},\end{cases}
$$


By Lemma 2.3, we get a contradiction. Thus

$$
(-\Delta)^{m-1} v \geq 0, \text { for } \quad r \geq 0 \text {. }
$$

If there exists $r_{4} \geq 0$ such that $(-\Delta)^{m-2} v\left(r_{4}\right)<0$, we have

$$
\Delta^{m-2} v(r) \leq \Delta^{m-2} v\left(r_{4}\right)<0, \quad \text { for } r \geq r_{4},
$$

since $m$ is an even number. From (2.12), we get

$$
v(r) \rightarrow-\infty, \quad \text { as } r \rightarrow+\infty,
$$

again a contradiction. Repeating the above process, we obtain, if there exists $r_{i} \geq 0$ such that $(-\Delta)^{i} v\left(r_{i}\right)<0$, then when $i$ is an odd number,

$$
v(r) \rightarrow+\infty, \quad \text { as } r \rightarrow+\infty,
$$

and when $i$ is an even number,

$$
v(r) \rightarrow-\infty, \quad \text { as } \quad r \rightarrow+\infty,
$$

in both cases, we get a contradiction by Lemma 2.3 and $v>0$. Thus

$$
(-\Delta)^{i} v \geq 0, \quad i=1, \ldots, m-1 \text {. }
$$

From (2.5) we have

$$
\Delta^{m} v=b(r) u^{q} v^{s} .
$$

Since $b(r)$ is non-decreasing and $u(r) \rightarrow+\infty$ as $r \rightarrow+\infty$, there exist $\tilde{c}>0$ and $\tilde{r}>0$ such that

Thus by Lemma 2.2,

$$
\Delta^{m} v \geq \tilde{c} v^{s}, \quad \text { for } \quad r \geq \tilde{r}
$$

$$
v \geq c r^{2 m} v^{s}(r), \quad \text { for } \quad r \geq 2 \tilde{r} .
$$

We get, when $0<s<1$,

$$
v(r) \geq c r^{2 m} \rightarrow+\infty, \quad \text { as } \quad r \rightarrow+\infty,
$$

a contradiction since $v(r)$ is non-increasing by (2.13); when $s=1$,

$$
1 \geq c r^{2 m}, \quad \text { for } \quad r \geq 2 \tilde{r} .
$$

again a contradiction. We complete the Step 1.

Step 2. We show that $(-\Delta)^{m-i} u \geq 0,(-\Delta)^{m-i} v \geq 0, i=2, \ldots, m-1$.

We prove this by induction on $i$. By step 1 . this is true for $i=1$. Assume that $(-\Delta)^{m-i+1} u \geq 0,(-\Delta)^{m-i+1} v \geq 0$. Set $w=(-\Delta)^{m-i} u$, suppose by contrary that there exists $\bar{r} \geq 0$ such that $w(\bar{r})<0$, we get

$$
w(r) \leq w(\bar{r})<0, \quad \text { for } \quad r \geq \bar{r},
$$

since $-\Delta w=(-\Delta)^{m-(i-1)} u \geq 0$. Again, we discuss two cases.

Case 1. $m-i$ is an even number.

From (2.16),

$$
\Delta^{m-i} u \leq w(\bar{r})<0
$$

this deduces that $u \rightarrow-\infty$ as $r \rightarrow+\infty$, a contradiction. 
Case 2. $m-i$ is an odd number.

From (2.16),

$$
\Delta^{m-i} u \geq-w(\bar{r})>0
$$

we get $u \rightarrow+\infty$ as $r \rightarrow+\infty$. We follow the same arguments in step 1 and lead to a contradiction. Thus we complete the proof of Proposition 2.7.

In order to prove Theorem 2.1 we make use of the following identity.

Lemma 2.8. Let $\Omega$ be a smooth bounded domain in $\mathbb{R}^{N}$, and $u, v \in C^{2 m}(\bar{\Omega})$, then

$$
\begin{array}{rl}
\int_{\Omega} \Delta^{m} & u(x, \nabla v) d x \\
= & \sum_{k=1}^{m} \int_{\partial \Omega}\left\{\frac{\partial\left(\Delta^{m-k} u\right)}{\partial n}\left(x, \nabla\left(\Delta^{k-1} v\right)\right)-\Delta^{m-k} u \frac{\partial\left(x, \nabla\left(\Delta^{k-1} v\right)\right)}{\partial n}\right\} d \sigma \\
& +2 \sum_{k=1}^{m} \sum_{i=1}^{m-k} \int_{\partial \Omega}\left\{\frac{\partial\left(\Delta^{m-k-i} u\right)}{\partial n} \Delta^{k+i-1} v-\Delta^{m-k-i} u \frac{\partial\left(\Delta^{k+i-1} v\right)}{\partial n}\right\} d \sigma \\
& +2 m \int_{\Omega} u \Delta^{m} v d x+\int_{\Omega} u\left(x, \nabla\left(\Delta^{m} v\right)\right) d x
\end{array}
$$

The proof is a straightforward application of Green's formula and can be found in [11].

Proof of Theorem 2.1. Set $u_{i}=(-\Delta)^{i} u, v_{i}=(-\Delta)^{i} v, i=1, \ldots, m-1$. From (2.1), we get

$$
\left\{\begin{array}{l}
\Delta u+u_{1}=0, \\
\Delta u_{i}+u_{i+1}=0, \quad i=1, \ldots, m-2, \\
\Delta u_{m-1}+u^{k} v^{p}=0, \\
\Delta v+v_{1}=0, \\
\Delta v_{i}+v_{i+1}=0, \quad i=1, \ldots, m-2, \\
\Delta v_{m-1}+u^{q} v^{s}=0,
\end{array}\right.
$$

By Lemma 2.2,

$$
\begin{aligned}
& u \geq c r^{2} u_{1}, \quad u_{i} \geq c r^{2} u_{i+1}, \quad i=1, \ldots, m-2, \quad u_{m-1} \geq c r^{2} u(r)^{k} v(r)^{p}, \\
& v \geq c r^{2} v_{1}, \quad v_{i} \geq c r^{2} v_{i+1}, \quad i=1, \ldots, m-2, \quad v_{m-1} \geq c r^{2} u(r)^{q} v(r)^{s} .
\end{aligned}
$$

From (2.19), (2.20),

$$
\left\{\begin{array}{l}
u(r) \leq c r^{\frac{-2 m(1-s+p)}{p q-(1-s)(1-k)}}, \\
v(r) \leq c r^{\frac{-2 m(1-k+q)}{p q-(1-s)(1-k)}}, \\
u_{i}(r) \leq c r^{-2 i+\frac{-2 m(1-s+p)}{p q-(1-s)(1-k)}}, \\
v_{i}(r) \leq c r^{-2 i+\frac{-2 i-2 m(1-k+q)}{p q-(1-s)(1-k)}} .
\end{array}\right.
$$


By Lemma 2.5,

$$
\left\{\begin{aligned}
-u^{\prime}(r) & \leq c r^{-1+\frac{-2 m(1-s+p)}{p q-(1-s)(1-k)}}, \\
-v^{\prime}(r) & \leq c r^{-1+\frac{-2 m(1-k+q)}{p q-(1-s)(1-k)}} \\
-u_{i}^{\prime}(r) & \leq c r^{-1-2 i+\frac{-2 m(1-s+p)}{p q-(1-s)(1-k)}} \\
-v_{i}^{\prime}(r) & \leq c r^{-1-2 i+\frac{-2 m(1-k+q)}{p q-(1-s)(1-k)}} .
\end{aligned}\right.
$$

Using (2.17) in $\Omega=B_{R}(0)$, we get

$$
\begin{aligned}
& \int_{0}^{R} r^{N} u^{k} v^{p} v^{\prime} d r \\
& =-\sum_{k=1}^{m}\left\{u_{m-k}^{\prime} v_{k-1}^{\prime} R-u_{m-k}\left(r v_{k-1}^{\prime}\right)\right\} R^{N-1} \\
& -2 \sum_{k=1}^{m} \sum_{i=1}^{m-k}\left(u_{m-k-i}^{\prime} v_{k+i-1}-u_{m-k-i} v_{k+i-1}^{\prime}\right) R^{N-1} \\
& +2 m \int_{0}^{R} r^{N-1} u^{q+1} v^{s} d r+\int_{0}^{R} u\left(u^{q} v^{s}\right)^{\prime} r^{N} d r \\
& =-\sum_{k=1}^{m}\left\{u_{m-k}^{\prime} v_{k-1}^{\prime} R-u_{m-k} v_{k} R+(N-2) u_{m-k} v_{k-1}^{\prime}\right\} R^{N-1} \\
& -2 \sum_{k=1}^{m} \sum_{i=1}^{m-k}\left(u_{m-k-i}^{\prime} v_{k+i-1}-u_{m-k-i} v_{k+i-1}^{\prime}\right) R^{N-1} \\
& +2 m \int_{0}^{R} r^{N-1} u^{q+1} v^{s} d r+\int_{0}^{R} u r^{N} d\left(u^{q} v^{s}\right) \\
& =-\sum_{k=1}^{m}\left\{u_{m-k}^{\prime} v_{k-1}^{\prime} R-u_{m-k} v_{k} R+(N-2) u_{m-k} v_{k-1}^{\prime}\right\} R^{N-1} \\
& -2 \sum_{k=1}^{m} \sum_{i=1}^{m-k}\left(u_{m-k-i}^{\prime} v_{k+i-1}-u_{m-k-i} v_{k+i-1}^{\prime}\right) R^{N-1} \\
& +2 m \int_{0}^{R} r^{N-1} u^{q+1} v^{s} d r+R^{N} u^{q+1} v^{s}-\int_{0}^{R} u^{q} v^{s} d\left(u r^{N}\right) \\
& =-\sum_{k=1}^{m}\left\{u_{m-k}^{\prime} v_{k-1}^{\prime} R-u_{m-k} v_{k} R+(N-2) u_{m-k} v_{k-1}^{\prime}\right\} R^{N-1} \\
& -2 \sum_{k=1}^{m} \sum_{i=1}^{m-k}\left(u_{m-k-i}^{\prime} v_{k+i-1}-u_{m-k-i} v_{k+i-1}^{\prime}\right) R^{N-1} \\
& +(2 m-N) \int_{0}^{R} r^{N-1} u^{q+1} v^{s} d r+R^{N} u^{q+1} v^{s}-\int_{0}^{R} r^{N} u^{q} u^{\prime} v^{s} d r .
\end{aligned}
$$


By (ii),

$$
\begin{array}{r}
-\sum_{k=1}^{m}\left\{u_{m-k}^{\prime} v_{k-1}^{\prime} R-u_{m-k} v_{k} R+(N-2) u_{m-k} v_{k-1}^{\prime}\right\} R^{N-1} \rightarrow 0, \quad \text { as } \quad R \rightarrow+\infty, \\
-2 \sum_{k=1}^{m} \sum_{i=1}^{m-k}\left(u_{m-k-i}^{\prime} v_{k+i-1}-u_{m-k-i} v_{k+i-1}^{\prime}\right) R^{N-1} \rightarrow 0, \quad \text { as } \quad R \rightarrow+\infty, \\
R^{N} u^{q+1} v^{s} \rightarrow 0, \quad \text { as } \quad R \rightarrow+\infty .
\end{array}
$$

Thus

$$
\begin{aligned}
& \int_{0}^{R} r^{N} u^{k} v^{p} v^{\prime} d r \\
& \quad=(2 m-N) \int_{0}^{R} r^{N-1} u^{q+1} v^{s} d r-\int_{0}^{R} r^{N} u^{q} u^{\prime} v^{s} d r+o(R),
\end{aligned}
$$

where $o(R)$ means infinitely small quantity.

Similarly we have

$$
\begin{aligned}
& \int_{0}^{R} r^{N} u^{q} v^{s} u^{\prime} d r \\
& \quad=(2 m-N) \int_{0}^{R} r^{N-1} v^{p+1} u^{k} d r-\int_{0}^{R} r^{N} u^{k} v^{p} v^{\prime} d r+o(R) .
\end{aligned}
$$

From (2.23), (2.24)

$$
\begin{aligned}
& \int_{0}^{R} r^{N} u^{k} v^{p} v^{\prime} d r+\int_{0}^{R} r^{N} u^{q} v^{s} u^{\prime} d r \\
& \quad=\left(m-\frac{N}{2}\right) \int_{0}^{R} r^{N-1} u^{q+1} v^{s} d r+\left(m-\frac{N}{2}\right) \int_{0}^{R} r^{N-1} v^{p+1} u^{k} d r+o(R) .
\end{aligned}
$$

Integrating the left-hand side of $(2.25)$ by parts on $(0, R)$ yields

$$
\begin{aligned}
\int_{0}^{R} r^{N} u^{k} v^{p} v^{\prime} d r+\int_{0}^{R} r^{N} u^{q} v^{s} u^{\prime} d r \\
=\frac{1}{p+1} R^{N} u^{k} v^{p+1}-\frac{N}{p+1} \int_{0}^{R} r^{N-1} u^{k} v^{p+1} d r \\
\quad-\frac{k}{p+1} \int_{0}^{R} r^{N} u^{k-1} u^{\prime} v^{p+1} d r+\frac{1}{q+1} R^{N} u^{q+1} v^{s}-\frac{N}{q+1} \int_{0}^{R} r^{N-1} u^{q+1} v^{s} d r \\
\quad-\frac{s}{q+1} \int_{0}^{R} r^{N} u^{q+1} v^{\prime} v^{s-1} d r .
\end{aligned}
$$


Using (ii), we get

$$
\begin{aligned}
\int_{0}^{R} r^{N} u^{k} v^{p} v^{\prime} d r+\int_{0}^{R} r^{N} u^{q} v^{s} u^{\prime} d r \\
=-\frac{N}{p+1} \int_{0}^{R} r^{N-1} u^{k} v^{p+1} d r-\frac{k}{p+1} \int_{0}^{R} r^{N} u^{k-1} u^{\prime} v^{p+1} d r \\
\quad-\frac{N}{q+1} \int_{0}^{R} r^{N-1} u^{q+1} v^{s} d r-\frac{s}{q+1} \int_{0}^{R} r^{N} u^{q+1} v^{\prime} v^{s-1} d r+o(R) .
\end{aligned}
$$

By Lemma 2.5,

$$
\begin{aligned}
\int_{0}^{R} r^{N} u^{k} v^{p} v^{\prime} d r+\int_{0}^{R} r^{N} u^{q} v^{s} u^{\prime} d r \\
\leq-\frac{N}{p+1} \int_{0}^{R} r^{N-1} u^{k} v^{p+1} d r+\frac{k(N-2 m)}{p+1} \int_{0}^{R} r^{N-1} u^{k} v^{p+1} d r \\
\quad-\frac{N}{q+1} \int_{0}^{R} r^{N-1} u^{q+1} v^{s} d r+\frac{s(N-2 m)}{q+1} \int_{0}^{R} r^{N-1} u^{q+1} v^{s} d r+o(R) \\
=\frac{k(N-2 m)-N}{p+1} \int_{0}^{R} r^{N-1} u^{k} v^{p+1} d r \\
\quad+\frac{s(N-2 m)-N}{q+1} \int_{0}^{R} r^{N-1} u^{q+1} v^{s} d r+o(R) .
\end{aligned}
$$

From (2.25),

$$
\begin{aligned}
& \left(m-\frac{N}{2}-\frac{s(N-2 m)-N}{q+1}\right) \int_{0}^{R} r^{N-1} u^{q+1} v^{s} d r \\
& \quad+\left(m-\frac{N}{2}-\frac{k(N-2)-N}{p+1}\right) \int_{0}^{R} r^{N-1} v^{p+1} u^{k} d r+o(R) \leq 0 .
\end{aligned}
$$

Using (ii), we get

$$
\int_{0}^{R} r^{N-1} u^{q+1} v^{s} d r<+\infty, \quad \int_{0}^{R} r^{N-1} v^{p+1} u^{k} d r<+\infty .
$$

From (2.23), (2.24),

$$
\int_{0}^{R} r^{N-1} u^{q+1} v^{s} d r=\int_{0}^{R} r^{N-1} v^{p+1} u^{k} d r+o(R) .
$$

Passing to the limit $R \rightarrow+\infty$ in the (2.28),

$$
\left\{2 m-N-\frac{s(N-2 m)-N}{q+1}-\frac{k(N-2 m)-N}{p+1}\right\} \int_{0}^{+\infty} r^{N-1} v^{p+1} u^{k} d r \leq 0,
$$

which contradicts (ii), and this concludes the proof of Theorem 2.1. 


\section{A priori estimates}

In this section we follow the approach suggested in [3] to prove a priori estimates for positive radial solutions of the polyharmonic system (1.1).

Lemma 3.1. Let $u \in C^{1}\left[r_{0},+\infty\right) \cap C^{2}\left(r_{0},+\infty\right)$ be a nonnegative solution of

$$
-\left(r^{N-1} u^{\prime}(r)\right)=r^{N-1} f(r), \quad \text { in }\left[r_{0},+\infty\right),
$$

where $f \in C\left(r_{0},+\infty\right)$ and is nonnegative, $r_{0} \geq 0$. Assume that

$$
u\left(r_{0}\right)>0, \text { and } u^{\prime}\left(r_{0}\right)<0 .
$$

Then

$$
u(r)>0 \quad \text { and } \quad u^{\prime}(r) \leq 0, \text { for } r \geq r_{0} .
$$

This lemma is a simple version of Lemma 2.1 in [3].

Proposition 3.2. Under the assumptions of Theorem 1.1, if $(u, v)$ denotes any positive radial solution of the polyharmonic system (1.1), then there exists a constant $C$ independent of $u$ and $v$ such that

$$
\begin{aligned}
& \|u\|_{\infty} \leq C, \quad\left\|(-\Delta)^{i} u\right\|_{\infty} \leq C, \quad\|v\|_{\infty} \leq C, \quad\left\|(-\Delta)^{i} v\right\|_{\infty} \leq C, \\
& i=1, \ldots, m-1
\end{aligned}
$$

where $\|\cdot\|_{\infty}$ is the norm in $C([0, R])$.

Proof. We show that $(-\Delta)^{i} u \geq 0,(-\Delta)^{i} v \geq 0, i=1, \ldots, m-1$. Set $w=$ $(-\Delta)^{m-1} u$, it is easy to see $-\Delta w=(-\Delta)^{m} u \geq 0$. Multiplying the inequality by $r^{N-1}$ and integrate the resulting inequality, we have $\frac{d w}{d r} \leq 0$, thus $w$ is nonincreasing. By the boundary condition $(-\Delta)^{m-1} u(R)=0$, we have $(-\Delta)^{m-1} u \geq$ 0 , for $r \in[0, R]$. Similarly, we obtain $(-\Delta)^{i} u \geq 0,(-\Delta)^{i} v \geq 0$, for $r \in[0, R], i=$ $1, \ldots, m-1$.

Set $u_{i}=(-\Delta)^{i} u, v_{i}=(-\Delta)^{i} v, i=1, \ldots, m-1$, we rewrite the polyharmonic system (1.1) as

$$
\left\{\begin{array}{l}
\Delta u+u_{1}=0, \quad \text { in } B_{R}, \\
\Delta u_{i}+u_{i+1}=0, \quad i=1, \ldots, m-2, \quad \text { in } B_{R}, \\
\Delta u_{m-1}+u^{k} v^{p}=0, \quad \text { in } B_{R}, \\
\Delta v+v_{1}=0, \quad \text { in } B_{R}, \\
\Delta v_{i}+v_{i+1}=0, \quad i=1, \ldots, m-2, \quad \text { in } B_{R}, \\
\Delta v_{m-1}+u^{q} v^{s}=0 \text { in } B_{R}, \\
u=u_{i}=v=v_{i}=0, \quad \text { on } \partial B_{R}, \quad i=1, \ldots, m-1 .
\end{array}\right.
$$

We argue by contradiction. Thus we assume that there is a sequence

$$
\left\{\left(u_{n}, u_{1, n}, \cdots, u_{m-1, n}, v_{n}, v_{1, n}, \cdots, v_{m-1, n}\right)\right\}
$$

of positive solutions of system (3.2) such that

$$
\lim _{n \rightarrow \infty}\left(t_{n}+\sum_{i=1}^{m-1} t_{i, n}+s_{n}+\sum_{i=1}^{m-1} s_{i, n}\right)=+\infty,
$$


where

$$
\begin{aligned}
& t_{n}=\sup _{r \in[0, R]} u_{n}(r)=u_{n}(0), \quad t_{i, n}=\sup _{r \in[0, R]} u_{i, n}(r)=u_{i, n}(0), \quad i=1, \ldots, m-1, \\
& s_{n}=\sup _{r \in[0, R]} v_{n}(r)=v_{n}(0), \quad s_{i, n}=\sup _{r \in[0, R]} v_{i, n}(r)=v_{i, n}(0), \quad i=1, \ldots, m-1 .
\end{aligned}
$$

Let us define

$$
\lambda=\frac{2 m(p+1-s)}{p q-(1-k)(1-s)}, \quad \mu=\frac{2 m(q+1-k)}{p q-(1-k)(1-s)} .
$$

Set

$$
\gamma_{n}=\left(t_{n}\right)^{\frac{1}{\lambda}}+\sum_{i=1}^{m-1}\left(t_{i, n}\right)^{\frac{1}{\lambda+2 i}}+\left(s_{n}\right)^{\frac{1}{\mu}}+\sum_{i=1}^{m-1}\left(s_{i, n}\right)^{\frac{1}{\mu+2 i}}
$$

then $\gamma_{n} \rightarrow+\infty$, as $n \rightarrow+\infty$. Put $y=\gamma_{n} r$,

$$
\begin{aligned}
w_{n}(y) & =\frac{1}{\gamma_{n}^{\lambda}} u_{n}(r), \quad w_{i, n}(y)=\frac{1}{\gamma_{n}^{\lambda+2 i}} u_{i, n}(r), \quad i=1, \ldots, m-1, \\
z_{n}(y) & =\frac{1}{\gamma_{n}^{\mu}} v_{n}(r), \quad z_{i, n}(y)=\frac{1}{\gamma_{n}^{\mu+2 i}} v_{i, n}(r), \quad i=1, \ldots, m-1 .
\end{aligned}
$$

We have that

$$
\begin{array}{r}
w_{n}(y) \leq 1, \quad w_{i, n}(y) \leq 1, \quad z_{n}(y) \leq 1, \quad z_{i, n}(y) \leq 1, \quad y \in\left[0, R \gamma_{n}\right] \\
i=1, \ldots, m-1
\end{array}
$$

and

$$
\begin{aligned}
w_{n}\left(R \gamma_{n}\right) & =w_{i, n}\left(R \gamma_{n}\right)=z_{n}\left(R \gamma_{n}\right)=z_{i, n}\left(R \gamma_{n}\right)=0, \quad i=1, \ldots, m-1, \\
w_{n}^{\prime}(0) & =w_{i, n}^{\prime}(0)=z_{n}^{\prime}(0)=z_{i, n}^{\prime}(0)=0, \quad i=1, \ldots, m-1 .
\end{aligned}
$$

By the definition of $\lambda, \mu$,

$$
p \mu+\lambda(k-1)-2 m=q \lambda+\mu(s-1)-2 m=0 .
$$

Then

or in short

$$
\left\{\begin{array}{l}
\Delta w_{n}+w_{1, n}=0, \\
\Delta w_{i, n}+w_{i+1, n}=0, \quad i=1, \ldots, m-2, \\
\Delta w_{m-1, n}+\gamma_{n}^{p \mu+\lambda(k-1)-2 m} w_{n}^{k} z_{n}^{p}=0 \\
\Delta z_{n}+z_{1, n}=0 \\
\Delta z_{i, n}+z_{i+1, n}=0, \\
\Delta z_{m-1, n}+\gamma_{n}^{q \lambda+\mu(s-1)-2 m} w_{n}^{q} z_{n}^{s}=0 .
\end{array}\right.
$$

$$
\Delta W_{n}+f\left(W_{n}\right)=0
$$

where

$$
\begin{aligned}
W_{n} & =\left(w_{n}, w_{1, n}, \ldots, w_{m-1, n}, z_{n}, z_{1, n}, \ldots, z_{m-1, n}\right) \\
f\left(W_{n}\right) & =\left(w_{1, n}, \ldots, w_{m-1, n}, w_{n}^{k} z_{n}^{p}, z_{1, n}, \ldots, z_{m-1, n}, w_{n}^{q} z_{n}^{s}\right) .
\end{aligned}
$$


Since

$$
\begin{aligned}
& w_{n}(0)=\frac{u_{n}(0)}{\gamma_{n}^{\lambda}}, \quad w_{i, n}(0)=\frac{u_{i, n}(0)}{\gamma_{n}^{\lambda+2 i}}, \quad i=1, \ldots, m-1, \\
& z_{n}(0)=\frac{v_{n}(0)}{\gamma_{n}^{\mu}}, \quad z_{i, n}(0)=\frac{v_{i, n}(0)}{\gamma_{n}^{\mu+2 i}}, \quad i=1, \ldots, m-1,
\end{aligned}
$$

we have that

$$
w_{n}^{\frac{1}{\lambda}}(0)+\sum_{i=1}^{m-1} w_{i, n}^{\frac{1}{\lambda+2 i}}(0)+z_{n}^{\frac{1}{\mu}}(0)+\sum_{i=1}^{m-1} z_{i, n}^{\frac{1}{\mu+2 i}}(0)=1 .
$$

Thus $\left\{w_{n}(0)\right\},\left\{w_{i, n}(0)\right\},\left\{z_{n}(0)\right\},\left\{z_{i, n}(0)\right\}$ are bounded sequences of real number. Passing to subsequence if necessary we can assume that

$$
\begin{aligned}
& w_{n}(0) \rightarrow \hat{w}_{0}, \quad w_{i, n}(0) \rightarrow \hat{w}_{i, 0}, \quad z_{n}(0) \rightarrow \hat{z}_{0}, \quad z_{i, n}(0) \rightarrow \hat{z}_{i, 0}, \\
& \text { as } \quad n \rightarrow \infty, \quad i=1, \ldots, m-1,
\end{aligned}
$$

and hence

$$
\hat{w}_{0}+\sum_{i=1}^{m-1} \hat{w}_{i, 0}+\hat{z}_{0}+\sum_{i=1}^{m-1} \hat{z}_{i, 0}=1 .
$$

For any $\bar{R} \in\left(0, R \gamma_{n}\right),\left\{w_{n}\right\},\left\{w_{i, n}\right\},\left\{z_{n}\right\},\left\{z_{i, n}\right\}$ are uniformly bounded sequences in $C([0, \bar{R}])$. We claim $\left\{w_{n}\right\},\left\{w_{i, n}\right\},\left\{z_{n}\right\},\left\{z_{i, n}\right\}$ are equicontinuous in $C([0, R])$. Multiplying $(3.11)_{1}$ by $w_{n}^{\prime},(3.11)_{2}$ by $w_{i, n}^{\prime},(3.11)_{3}$ by $w_{m-1, n}^{\prime},(3.11)_{4}$ by $z_{n}^{\prime}$, $(3.11)_{5}$ by $z_{i, n}^{\prime},(3.11)_{6}$ by $z_{m-1, n}^{\prime}$, we get

$$
\frac{1}{2}\left(\left(W_{n}^{\prime}\right)^{2}\right)^{\prime}+\frac{N-1}{y}\left(W_{n}^{\prime}\right)^{2}+g\left(W_{n}\right)=0,
$$

where

$$
g\left(W_{n}\right)=\left(w_{1, n} w_{n}^{\prime}, w_{2, n} w_{1, n}^{\prime}, \ldots, w_{n}^{k} z_{n}^{p} w_{m-1, n}^{\prime}, z_{1, n} z_{n}^{\prime}, z_{2, n} z_{1, n}^{\prime}, \ldots, w_{n}^{q} z_{n}^{s} z_{m-1, n}^{\prime}\right) .
$$

Since $w_{n}^{\prime}, w_{i, n}^{\prime}, z_{n}^{\prime}, z_{i, n}^{\prime} \leq 0$, it follows from (3.8) that

$$
\frac{1}{2}\left(\left(W_{n}^{\prime}\right)^{2}\right)^{\prime}+W_{n}^{\prime} \leq 0 .
$$

Integrating the above inequality from 0 to $y$, we have

$$
\frac{1}{2}\left(W_{n}^{\prime}(y)\right)^{2}+\int_{0}^{y} W_{n}^{\prime}(t) d t \leq 0 .
$$

and hence

$$
\begin{aligned}
\left|w_{n}^{\prime}(y)\right| \leq c, \quad\left|w_{i, n}^{\prime}(y)\right| \leq c, \quad\left|z_{n}^{\prime}(y)\right| \leq c, \quad & \left|z_{i, n}^{\prime}(y)\right| \leq c, \\
& i=1, \ldots, m-1,
\end{aligned}
$$

uniformly in $n$, since $\left\{w_{n}^{\prime}\right\},\left\{w_{i, n}^{\prime}\right\},\left\{z_{n}^{\prime}\right\},\left\{z_{i, n}^{\prime}\right\}$ are uniformly bounded sequences in $C([0, \bar{R}])$. From $(3.15)$, we obtain the equicontinuity of the sequences $\left\{w_{n}^{\prime}\right\},\left\{w_{i, n}^{\prime}\right\}$, 
$\left\{z_{n}^{\prime}\right\},\left\{z_{i, n}^{\prime}\right\}$. Thus, by Ascoli-Arzela Theorem, and passing to subsequence if necessary, we can assume that

$$
w_{n} \rightarrow \hat{w}, \quad w_{i, n} \rightarrow \hat{w}_{i}, \quad z_{n} \rightarrow \hat{z}, \quad z_{i, n} \rightarrow \hat{z}_{i}
$$

$$
\text { as } n \rightarrow \infty, \quad i=1, \ldots, m-1 \text {, }
$$

in $C([0, \bar{R}])$. From $(3.11)$,

$$
W_{n}(0)-W_{n}(y)=\int_{0}^{y} \frac{d \xi}{\xi^{N-1}} \int_{0}^{\xi} \eta^{N-1} h\left(W_{n}(\eta)\right) d \eta
$$

where

$$
h\left(W_{n}\right)=\left(w_{1, n}, w_{2, n}, \ldots, w_{m-1, n}, w_{n}^{k} z_{n}^{p}, z_{1, n}, z_{2, n}, \ldots, z_{m-1, n}, w_{n}^{q} z_{n}^{s}\right) .
$$

By the Dominated Convergence Theorem, letting $n \rightarrow \infty$ in (3.17), we have

$$
\hat{W}(0)-\hat{W}(y)=\int_{0}^{y} \frac{d \xi}{\xi^{N-1}} \int_{0}^{\xi} \eta^{N-1} h(\hat{W})(\eta) d \eta,
$$

which imply that $\hat{w} \geq 0, \hat{w}_{i} \geq 0, \hat{z} \geq 0, \hat{z}_{i} \geq 0$ belong to $C^{1}([0, \bar{R}]) \cap C^{2}((0, \bar{R}])$ and satisfy

$$
-\left(y^{N-1} \hat{W}^{\prime}\right)^{\prime}=y^{N-1} h(\hat{W}),
$$

for $y \in[0, \bar{R}]$, and

$$
\hat{w}^{\prime}(0)=\hat{w}_{i}^{\prime}(0)=\hat{z}^{\prime}(0)=\hat{z}_{i}^{\prime}(0)=0, \quad i=1, \ldots, m-1 .
$$

We claim that $\hat{w}, \hat{w}_{i}, \hat{z}, \hat{z}_{i}$ can be extended to $[0,+\infty)$. In fact we can repeat the above argument on an interval $\left[0, R^{*}\right], R^{*}>\bar{R}$, for the convergent sequences $\hat{w}, \hat{w}_{i}, \hat{z}, \hat{z}_{i}$ on $[0, \bar{R}]$. We get functions $\tilde{w}, \tilde{w}_{i}, \tilde{z}, \tilde{z}_{i}$, solutions of $(3.19)$ on $\left[0, R^{*}\right]$ that satisfy

$$
\begin{aligned}
& \hat{w}(y)=\tilde{w}(y), \quad \hat{w}_{i}(y)=\tilde{w}_{i}(y), \quad \hat{z}(y)=\tilde{z}(y), \quad \hat{z}_{i}(y)=\tilde{z}_{i}(y), \\
& \text { for all } y \in[0, \bar{R}], \quad i=1, \ldots, m-1 .
\end{aligned}
$$

Clearly, $\hat{w}, \hat{w}_{i}, \hat{z}, \hat{z}_{i}$ can be extended to $[0,+\infty)$ as solutions of (3.19) that satisfy $(3.20)$, and $\hat{w}(y) \geq 0, \hat{w}_{i}(y) \geq 0, \hat{z}(y) \geq 0, \hat{z}_{i}(y) \geq 0$ for $y \in[0,+\infty)$, hence the claim follows.

We now show that

$$
\hat{w}(0)>0, \quad \hat{w}_{i}(0)>0, \quad \hat{z}(0)>0, \quad \hat{z}_{i}(0)>0, \quad i=1, \ldots, m-1 .
$$

Without loss generality, suppose that $\hat{w}(0)=0$. Since $\hat{w}(y) \geq 0$ and $\hat{w}^{\prime}(y) \leq 0$, it follows that $\hat{w} \equiv 0$. From (3.19), we have

$$
\hat{w}_{i} \equiv 0, \quad i=1, \ldots, m-1 .
$$

From the last equation of (3.19), we get $-\Delta \hat{z}_{m-1}=0$, hence $\hat{z}_{m-1}$ is constant since $\hat{z}_{m-1}$ is a nonnegative harmonic function. By the proof of Theorem 1.1, $\hat{z}_{m-1}$ has 
decay property, this implies that $\hat{z}_{m-1} \equiv 0$. Similarly, we deduce that $\hat{z}=\hat{z}_{i} \equiv 0$, $i=1, \ldots, m-2$. Thus

$$
\hat{w}(0)+\sum_{i=1}^{m-1} \hat{w}_{i}(0)+\hat{z}(0)+\sum_{i=1}^{m-1} \hat{z}_{i}(0)=0,
$$

which contradicts with (3.13) and we proved (3.21).

By Lemma 3.1, we have

$$
\begin{aligned}
\hat{w}(y)>0, \quad \hat{w}_{i}(y)>0, \quad \hat{z}(y)>0, \quad \hat{z}_{i}(y)>0, \\
\quad \text { for all } \quad y \in[0,+\infty), \quad i=1, \ldots, m-1 .
\end{aligned}
$$

Thus we obtain a positive radial classical solution of the following system

$$
\left\{\begin{aligned}
(-\Delta)^{m} u & =u^{k} v^{p}, \\
(-\Delta)^{m} v & =u^{q} v^{s},
\end{aligned} \quad \text { in } \quad \mathbb{R}^{N} .\right.
$$

This is impossible in view of Theorem 2.1 and the proof is completed.

Proposition 3.3. Consider the polyharmonic system

$$
\left\{\begin{array}{l}
(-\Delta)^{m} u=(u+\tau)^{k}\left(v+\tau^{\sigma}\right)^{p}, \quad \text { in } B_{R}, \\
(-\Delta)^{m} v=(u+\tau)^{q}\left(v+\tau^{\sigma}\right)^{s}, \quad \text { in } B_{R}, \\
u=(-\Delta) u=\cdots=(-\Delta)^{m-1} u=0, \quad \text { on } \partial B_{R}, \\
v=(-\Delta) v=\cdots=(-\Delta)^{m-1} v=0, \quad \text { on } \partial B_{R},
\end{array}\right.
$$

where $\tau \in(0,+\infty)$ is a parameter, $\sigma$ satisfies

$$
\left\{\begin{array}{l}
1<k+\sigma p \\
(1-s) \sigma<q .
\end{array}\right.
$$

If (3.24) possesses a positive radial solution $(u, v)$, then there is a constant $M>0, M$ independent of $u$ and $v$, such that $0 \leq \tau \leq M$.

Proof. Set $u_{i}=(-\Delta)^{i} u, v_{i}=(-\Delta)^{i} v, i=1, \ldots, m-1$, we rewrite the polyharmonic system (3.24) as

$$
\left\{\begin{array}{l}
\Delta u+u_{1}=0, \\
\Delta u_{i}+u_{i+1}=0, \quad i=1, \ldots, m-2, \\
\Delta u_{m-1}+(u+\tau)^{k}\left(v+\tau^{\sigma}\right)^{p}=0, \\
\Delta v+v_{1}=0, \\
\Delta v_{i}+v_{i+1}=0, \quad i=1, \ldots, m-2, \\
\Delta v_{m-1}+(u+\tau)^{q}\left(v+\tau^{\sigma}\right)^{s}=0 .
\end{array}\right.
$$

From the third equation and the last equation in (3.26), we have

$$
\begin{aligned}
& -u_{m-1}^{\prime} \geq c r(u+\tau)^{k}\left(v+\tau^{\sigma}\right)^{p} \geq c r \tau^{k+\sigma p}, \\
& -v_{m-1}^{\prime} \geq c r(u+\tau)^{q}\left(v+\tau^{\sigma}\right)^{s} \geq c r \tau^{q+\sigma s} .
\end{aligned}
$$

Integrating (3.27) and (3.28) from 0 to $R$, we obtain that

$$
u_{m-1}(0) \geq c R^{2} \tau^{k+\sigma p}, \quad v_{m-1}(0) \geq c R^{2} \tau^{q+\sigma s} .
$$


Integrating (3.27) from $r$ to $R$,

$$
u_{m-1}(r) \geq c \tau^{k+\sigma p}\left(R^{2}-r^{2}\right) .
$$

Again from (3.26),

$$
-u_{m-2}^{\prime} \geq c r u_{m-1},
$$

integrating the above inequality from 0 to $R$, we have

$$
\begin{aligned}
u_{m-2}(0) & \geq \int_{0}^{R} c r u_{m-1}(r) d r \\
& \geq \int_{0}^{R} c r \tau^{k+\sigma+p}\left(R^{2}-r^{2}\right) d r \\
& =\frac{1}{4} c R^{4} \tau^{k+\sigma p} .
\end{aligned}
$$

Repeating the above process, we obtain

$$
u(0) \geq c \tau^{k+\sigma p} .
$$

Similarly, we have

$$
v(0) \geq c \tau^{q+\sigma s} .
$$

Suppose now that proposition is not true. Then there is a sequence $\left\{\tau_{n}\right\}, \tau_{n} \rightarrow$ $+\infty$, as $n \rightarrow \infty$ such that for each $\tau_{n}$ there exist solutions $u_{n}, v_{n}$ of (3.24). Denoting as in Proposition 3.2,

$$
\begin{aligned}
& t_{n}=\sup _{r \in[0, R]} u_{n}(r)=u_{n}(0), \quad t_{i, n}=\sup _{r \in[0, R]} u_{i, n}(r)=u_{i, n}(0), \quad i=1, \ldots, m-1, \\
& s_{n}=\sup _{r \in[0, R]} v_{n}(r)=v_{n}(0), \quad s_{i, n}=\sup _{r \in[0, R]} v_{i, n}(r)=v_{i, n}(0), \quad i=1, \ldots, m-1 .
\end{aligned}
$$

and

$$
\gamma_{n}=\left(t_{n}\right)^{\frac{1}{\lambda}}+\sum_{i=1}^{m-1}\left(t_{i, n}\right)^{\frac{1}{\lambda+2 i}}+\left(s_{n}\right)^{\frac{1}{\mu}}+\sum_{i=1}^{m-1}\left(s_{i, n}\right)^{\frac{1}{\mu+2 i}},
$$

then from (3.31), $t_{n} \rightarrow+\infty$, and hence $\gamma_{n} \rightarrow+\infty$ as $n \rightarrow+\infty$. If now as in Proposition 3.2 we make the change of variables $y, w_{n}(y), w_{i, n}(y), z_{n}(y), z_{i, n}(y)$ indicated in (3.6) and (3.7), we have

$$
\left\{\begin{array}{l}
\Delta w_{n}+w_{1, n}=0, \\
\Delta w_{i, n}+w_{i+1, n}=0, \quad i=1, \ldots, m-2, \\
\Delta w_{m-1, n}+\left(w_{n}+\frac{\tau_{n}}{\gamma_{n}^{\lambda}}\right)^{k}\left(z_{n}+\frac{\tau_{n}^{\sigma}}{\gamma_{n}^{\mu}}\right)^{p}=0 \\
\Delta z_{n}+z_{1, n}=0, \\
\Delta z_{i, n}+z_{i+1, n}=0, \\
\Delta z_{m-1, n}+\left(w_{n}+\frac{\tau_{n}}{\gamma_{n}^{\lambda}}\right)^{q}\left(z_{n}+\frac{\tau_{n}^{\sigma}}{\gamma_{n}^{\mu}}\right)^{s}=0 .
\end{array}\right.
$$

Let $\bar{R}$ be a positive number as in Proposition 3.2 and consider the restriction of $w_{n}, w_{i, n}, z_{n}, z_{i, n}$ to $[0, \bar{R}]$, which we denote again by $w_{n}, w_{i, n}, z_{n}, z_{i, n}$. Repeating the argument of Proposition 3.2, we have that $w_{n}, w_{i, n}, z_{n}, z_{i, n}$ will meet the condition 
of Ascoli-Arzela Theorem if we can show from $(3.33)_{3}$ that the sequence $\left\{w_{m-1, n}\right\}$ is bounded.

Multiplying $(3.33)_{3}$ by $w_{m-1, n}^{\prime}$ we obtain

$$
\frac{1}{2}\left(\left(w_{m-1, n}^{\prime}\right)^{2}\right)^{\prime}+\left(w_{n}+\frac{\tau_{n}}{\gamma_{n}^{\lambda}}\right)^{p}\left(z_{n}+\frac{\tau_{n}^{\sigma}}{\gamma_{n}^{\mu}}\right) w_{m-1}^{\prime} \leq 0,
$$

which implies that

$$
\frac{1}{2}\left(\left(w_{m-1, n}^{\prime}\right)^{2}\right)^{\prime}+\left(1+\frac{\tau_{n}}{\gamma_{n}^{\lambda}}\right)^{k}\left(1+\frac{\tau_{n}^{\sigma}}{\gamma_{n}^{\mu}}\right)^{p} w_{m-1}^{\prime} \leq 0 .
$$

Similarly we have

$$
\frac{1}{2}\left(\left(z_{m-1, n}^{\prime}\right)^{2}\right)^{\prime}+\left(1+\frac{\tau_{n}}{\gamma_{n}^{\lambda}}\right)^{q}\left(1+\frac{\tau_{n}^{\sigma}}{\gamma_{n}^{\mu}}\right)^{s} z_{m-1}^{\prime} \leq 0 .
$$

Thus to bound the sequences $\left\{w_{m-1, n}^{\prime}\right\}$ and $\left\{z_{m-1, n}^{\prime}\right\}$ it is necessary to estimate $\frac{\tau_{n}}{\gamma_{n}^{\lambda}}$ and $\frac{\tau_{n}^{\sigma}}{\gamma_{n}^{\mu}}$. By (3.31) and (3.32), we have

$$
\tau_{n} \leq c t^{\frac{1}{k+\sigma p}} m, \quad \tau_{n} \leq c s^{\frac{1}{q+\sigma s}} m
$$

and thus

$$
\begin{aligned}
\frac{\tau_{n}}{\gamma_{n}^{\lambda}} & \leq \frac{c t^{\frac{1}{k+\sigma p}}}{\gamma_{n}^{\lambda}} \\
& \leq c \gamma_{n}^{\frac{\lambda}{k+\sigma p}-\lambda} \\
& =c \gamma_{n}^{\lambda\left(\frac{1}{k+\sigma p}-1\right)} \rightarrow 0, \text { as } n \rightarrow+\infty,
\end{aligned}
$$

since by $(3.25), \frac{1}{k+\sigma p}-1<0$. Similarly,

$$
\begin{aligned}
\frac{\tau_{n}^{\sigma}}{\gamma_{n}^{\mu}} & \leq \frac{c s^{\frac{\sigma}{q+\sigma s}}}{\gamma_{n}^{\mu}} \\
& \leq c \gamma_{n}^{\frac{\sigma \mu}{q+\sigma s}-\mu} \\
& =c \gamma_{n}^{\mu\left(\frac{\sigma}{q+\sigma s}-1\right)} \rightarrow 0, \text { as } n \rightarrow+\infty .
\end{aligned}
$$

Thus (3.37) and (3.38) imply the boundedness of $\left\{w_{m-1, n}^{\prime}\right\}$ and $\left\{z_{m-1, n}^{\prime}\right\}$.

The rest of the argument is exactly the same as that of Proposition 3.2. Thus we complete the proof of Proposition 3.3.

\section{Existence}

With the help of Proposition 3.2 and 3.3, we are able to use degree theory argument to prove Theorem 1.1.

The following fixed point theorem on a cone is due to deFueiredo, Lions and Naussbaum [6], which is a modified version of a theorem of Krasnosel'skii [9]. 
Theorem 4.1. Let $\mathcal{C}$ be a cone in a Banach space $X$ and $\mathfrak{T}: \mathcal{C} \rightarrow \mathcal{C}$ a compact mapping such that $\mathfrak{T}(0)=0$. Assume that there real numbers $0<\rho<R$ and $t_{0}>0$ such that

(i) $x \neq t \mathfrak{T}(x)$ for $0 \leq t \leq 1$ and $x \in \mathcal{C},\|x\|=\rho$, and

(ii) There exists a compact mapping $\mathfrak{H}: \overline{B_{R}} \times[0,+\infty) \rightarrow \mathcal{C}\left(\right.$ where $B_{r}=\{x \in \mathcal{C}$ : $\|x\|<r\})$ such that

(a) $\mathfrak{H}(x, 0)=\mathfrak{T}(x)$ for $\|x\|=R$,

(b) $\mathfrak{H}(x, t) \neq x$ for $\|x\|=R$ and $t \geq 0$

(c) $\mathfrak{H}(x, t)=x$ has no solution $x \in \overline{B_{R}}$ for $t \geq t_{0}$.

Then

$$
i_{\mathcal{C}}\left(\mathfrak{T}, B_{\rho}\right)=1, \quad i_{\mathcal{C}}\left(\mathfrak{T}, B_{R}\right)=0, \quad i_{\mathcal{C}}(\mathfrak{T}, \mathcal{U})=-1,
$$

where $\mathcal{U}=\{x \in \mathcal{C}: \rho<\|x\|<R\}$, and $i_{\mathcal{C}}$ denote the Leray-Schauder index.

Let us introduce the Banach space

$$
\begin{array}{r}
X=\left\{U=\left(u, u_{1}, \ldots, u_{m-1}, v, v_{1}, \ldots, v_{m-1}\right): u \in C([0, R]), u_{i} \in C([0, R]),\right. \\
\left.v \in C([0, R]), v_{i} \in C([0, R]), i=1, \ldots, m-1\right\},
\end{array}
$$

with the norm

$$
|U|=\|u\|_{\infty}+\sum_{i=1}^{m-1}\left\|u_{i}\right\|_{\infty}+\|v\|_{\infty}+\sum_{i=1}^{m-1}\left\|v_{i}\right\|_{\infty} .
$$

Let us denote by $\mathcal{C}$ the cone of nonnegative functions

$$
\begin{aligned}
\mathcal{C}=\left\{U \in X: u(r) \geq 0, u_{i}(r) \geq 0, v(r) \geq 0, v_{i}(r)\right. & \geq 0, \\
& \forall r \in[0, R], i=1, \ldots, m-1\} .
\end{aligned}
$$

Our system (1.1) is equivalent to $U=\mathfrak{T}(U)$ where

$$
\mathfrak{T}(U)=\left(\mathfrak{T}_{0}(U), \ldots, \mathfrak{T}_{2 m-1}(U)\right),
$$

where

$$
\begin{aligned}
\mathfrak{T}_{0}(U) & =\int_{r}^{R} \frac{d \xi}{\xi^{N-1}} \int_{0}^{\xi} \eta^{N-1} u_{1}(\eta) d \eta \\
\mathfrak{T}_{i}(U) & =\int_{r}^{R} \frac{d \xi}{\xi^{N-1}} \int_{0}^{\xi} \eta^{N-1} u_{i+1}(\eta) d \eta, \quad i=1, \ldots, m-2, \\
\mathfrak{T}_{m-1}(U) & =\int_{r}^{R} \frac{d \xi}{\xi^{N-1}} \int_{0}^{\xi} \eta^{N-1} u(\eta)^{k} v(\eta)^{p} d \eta, \\
\mathfrak{T}_{m}(U) & =\int_{r}^{R} \frac{d \xi}{\xi^{N-1}} \int_{0}^{\xi} \eta^{N-1} v_{1}(\eta) d \eta, \\
\mathfrak{T}_{m+i}(U) & =\int_{r}^{R} \frac{d \xi}{\xi^{N-1}} \int_{0}^{\xi} \eta^{N-1} v_{i+1}(\eta) d \eta, \quad i=1, \ldots, m-2, \\
\mathfrak{T}_{2 m-1}(U) & =\int_{r}^{R} \frac{d \xi}{\xi^{N-1}} \int_{0}^{\xi} \eta^{N-1} u(\eta)^{q} v(\eta)^{s} d \eta .
\end{aligned}
$$


It is easy to prove that $\mathfrak{T}$ maps $\mathcal{C}$ in $\mathcal{C}$ and $\mathfrak{T}$ is a compact operator.

The homotopy needed in this case is given by

$$
\mathfrak{H}(U, \tau)=T\left(\left(u+\tau, u_{1}, \ldots, u_{m-1}, v+\tau^{\sigma}, v_{1}, \ldots, v_{m-1}\right)\right),
$$

where $\sigma>0$ satisfying (3.25). Note that $\mathfrak{H}(U, \tau)$ is associated with a family of systems (3.26) parametrized by $\tau \geq 0$.

Proof of Theorem 1.1. We shall use Theorem 4.1. and divide the proof into two steps.

Step 1. Verification of condotion (ii).

Condition (a) clearly holds, since $\mathfrak{H}(0, U)=\mathfrak{T}(U), \forall U \in \mathcal{C}$.

Set $u_{i}=(-\Delta)^{i} u, v_{i}=(-\Delta)^{i} v, i=1, \ldots, m-1$, we rewrite the polyharmonic system (3.26) as

$$
\begin{aligned}
u(r) & =\int_{r}^{R} \frac{d \xi}{\xi^{N-1}} \int_{0}^{\xi} \eta^{N-1} u_{1}(\eta) d \eta \\
u_{i}(r) & =\int_{r}^{R} \frac{d \xi}{\xi^{N-1}} \int_{0}^{\xi} \eta^{N-1} u_{i+1}(\eta) d \eta, \quad i=1, \ldots, m-2, \\
u_{m-1}(r) & =\int_{r}^{R} \frac{d \xi}{\xi^{N-1}} \int_{0}^{\xi} \eta^{N-1}(u(\eta)+\tau)^{k}\left(v(\eta)+\tau^{\sigma}\right)^{p} d \eta \\
v(r) & =\int_{r}^{R} \frac{d \xi}{\xi^{N-1}} \int_{0}^{\xi} \eta^{N-1} v_{1}(\eta) d \eta, \\
v_{i}(r) & =\int_{r}^{R} \frac{d \xi}{\xi^{N-1}} \int_{0}^{\xi} \eta^{N-1} v_{i+1}(\eta) d \eta, \quad i=1, \ldots, m-2, \\
v_{m-1} & =\int_{r}^{R} \frac{d \xi}{\xi^{N-1}} \int_{0}^{\xi} \eta^{N-1}(u(\eta)+\tau)^{q}\left(v(\eta)+\tau^{\sigma}\right)^{s} d \eta
\end{aligned}
$$

Thus (3.26) is equivalent to

$$
U=\mathfrak{H}(U, \tau) .
$$

A slight modification of the proof Proposition 3.2 and Proposition 3.3 tell us that solutions of (4.2) are a priori bounded in $\mathcal{C}$. Hence there exists $R_{1}>0$ such that for any $\tau \in[0,+\infty)$ we have that

$$
U \neq \mathfrak{H}(U, \tau)
$$

with $|U|=R_{1}$. So (b) is verified.

If we take $\tau>M, M$ is in Proposition 3.3, it follows from this proposition that (c) is verified.

Step 2. Verification of condition (i).

We claim that there exists a $\bar{\rho}>0$ such that for any $0<\rho<\bar{\rho}$,

$$
U \neq \lambda \mathfrak{T}(U),
$$

for $\lambda \in[0,1]$ and any $U \in \mathcal{C}$ with $|U|=\rho$. 
Suppose the claim is no true. Then there exist $\left\{\rho_{n}\right\}, \rho_{n} \rightarrow 0^{+}$and sequences $\left\{\lambda_{n}\right\}, \lambda_{n} \in[0,1],\left\{U_{n}\right\}$ in $\mathcal{C}$ with $\left|U_{n}\right|=\rho_{n}$ such that

$$
U_{n}=\lambda_{n} \mathfrak{T}_{n},
$$

By (4.3), we have that $u_{n}, u_{i, n}, v_{n}, v_{i, n}$ satisfy

$$
\left\{\begin{array}{l}
u_{n}(0)=\int_{0}^{R} \frac{d \xi}{\xi^{N-1}} \int_{0}^{\xi} \lambda_{n} \eta^{N-1} u_{1, n}(\eta) d \eta \\
u_{i, n}(0)=\int_{0}^{R} \frac{d \xi}{\xi^{N-1}} \int_{0}^{\xi} \lambda_{n} \eta^{N-1} u_{i+1, n}(\eta) d \eta, \quad i=1, \ldots, m-2, \\
u_{m-1, n}(0)=\int_{0}^{R} \frac{d \xi}{\xi^{N-1}} \int_{0}^{\xi} \lambda_{n} \eta^{N-1} w_{n}^{k}(\eta) z_{n}^{p}(\eta) d \eta \\
v_{n}(0)=\int_{0}^{R} \frac{d \xi}{\xi^{N-1}} \int_{0}^{\xi} \lambda_{n} \eta^{N-1} v_{1, n}(\eta) d \eta \\
v_{i, n}(0)=\int_{0}^{R} \frac{d \xi}{\xi^{N-1}} \int_{0}^{\xi} \lambda_{n} \eta^{N-1} v_{i+1, n}(\eta) d \eta, \quad i=1, \ldots, m-2, \\
v_{m-1, n}(0)=\int_{0}^{R} \frac{d \xi}{\xi^{N-1}} \int_{0}^{\xi} \lambda_{n} \eta^{N-1} w_{n}^{q}(\eta) z_{n}^{s}(\eta) d \eta .
\end{array}\right.
$$

Noting that $\left\|u_{n}\right\|_{\infty},\left\|u_{i, n}\right\|_{\infty},\left\|v_{n}\right\|_{\infty},\left\|v_{n}\right\|_{\infty}$ can not be zero, from (4.4), we obtain

$$
\begin{aligned}
\left\|u_{n}\right\|_{\infty} & \leq c \lambda_{n}\left\|u_{1, n}\right\|_{\infty}, \\
\left\|u_{i, n}\right\|_{\infty} & \leq c \lambda_{n}\left\|u_{i+1, n}\right\|_{\infty}, \quad i=1, \ldots, m-2, \\
\left\|u_{m-1, n}\right\|_{\infty} & \leq c \lambda_{n}\left\|u_{n}\right\|_{\infty}^{k}\left\|v_{n}\right\|_{\infty}^{p}, \\
\left\|v_{n}\right\|_{\infty} & \leq c \lambda_{n}\left\|v_{1, n}\right\|_{\infty}, \\
\left\|v_{i, n}\right\|_{\infty} & \leq c \lambda_{n}\left\|v_{i+1, n}\right\|_{\infty}, \quad i=1, \ldots, m-2, \\
\left\|v_{m-1, n}\right\|_{\infty} & \leq c \lambda_{n}\left\|u_{n}\right\|_{\infty}^{q}\left\|v_{n}\right\|_{\infty}^{s},
\end{aligned}
$$

thus

$$
\left\|u_{n}\right\|_{\infty}^{1-k} \leq c \lambda_{n}^{m}\left\|v_{n}\right\|_{\infty}^{p}, \quad\left\|v_{n}\right\|_{\infty}^{1-s} \leq c \lambda_{n}^{m}\left\|u_{n}\right\|^{q},
$$

which imply that when $0 \leq k<1$ and $0 \leq s<1$,

$$
\begin{aligned}
1 & \leq c\left\|u_{n}\right\|^{\frac{p q}{(1-k)(1-s)}-1} \\
& \leq c \rho_{n}^{\frac{p q}{(1-k)(1-s)}-1},
\end{aligned}
$$

this leads us to a contradiction when $n \rightarrow \infty$. When $k=1$ or $s=1$, it is obvious to obtain a contradiction. Hence the claim is proved. Consequently (i) is verified. Thus we complete the proof of Theorem 1.1.

\section{References}

[1] T. Bartsch, Y. Guo, Existence and nonexistence results for critical growth polyharmonic elliptic systems, J. Diff. Eqs. 220 (2006), 531-543.

[2] Ph. Clément, J. Fleckinger, E. Mitidieri and F. de Thélin, Existence of positive solution for a nonvariational quasilinear elliptic system, J. Diff. Eqs. 166 (2000), 455-477.

[3] Ph. Clément, R. Manasevich and E. Mitidieri, Positive solution for a quasilinear system via blow-up, Comm. Part. Diff. Eqs. 18 (1993), 2071-2106. 
[4] D. G. de Figueiredo, Semilinear elliptic system, Nonlinear Funct. Anal. Appl. Diff. Equ., World Sci. Publishing, River Edge, NJ,1998, 122-152.

[5] D. G. de Figueiredo, P. L. Felmer, On superquadratic elliptic systems, Trans. Amer. Math. Soc. 343 (1994), 99-116.

[6] D. G. de Figueiredo, P. Lions, R. Naussbaum, A priori estimates and existence of positive solutions of semilinear elliptic equations, J. Math. Pures et Appl. 61 (1982), 41-63.

[7] B. Gidas, J. Spruck, A priori bounds for positive solutions of nonlinear elliptic equations, Comm. Part. Diff. Eqs. 6 (1981), 883-901.

[8] J. Hulshof, R. Van Der Vorst, Differential systems with strongly indefinite variational structure, J. Func. Anal. 114 (1993), 32-58.

[9] M. A. Krasnosel'skii, Fixed points of cone-compressing and cone-extending operators, Soviet. Math. Dolk. 1 (1960), 1285-1288.

[10] J. Liu, Y. Guo, Y. Zhang, Liouville-type theorems for polyharmonic system in $\mathbb{R}^{N}$, J. Diff. Eqs. 225 (2006), 685-709.

[11] J. Liu, Y. Guo, Y. Zhang, Existence of positive entire solutions for polyharmonic equations and systems, J. Partial Diff. Eqs. 19 (2006), 256-270.

$[12]$ L. A. Peletier, R. Van Der Vorst, Existence and nonexistence of positive solutions of nonlinear elliptic systems and the biharmonic equation, Diff. Int. Eq. 5 (1992), $747-767$

[13] M. A.S. Souto, A priori estimates and existence of positive solutions of nonlinear cooperative elliptic systems, Diff. Int. Eq. 8 (1995), 1245-1258.

[14] X. Xu, Uniqueness theorem for the entire positive solutions of biharmonic equations in $\mathbb{R}^{N}$, Proc. Royal. Soc. Edinburgh 130A (2000), 651-670.

[15] Y. Zhang, On the nonexistence of positive solutions of polyharmonic systems in $\mathbb{R}^{N}$, Nonlinear Anal. 68 (2008), 3454-3464.

[16] Y. Zhang, Liouville type theorems for polyharmonic systems, $\mathrm{PhD}$ thesis, Peking University (2006).

[17] H. Zou, A priori estimates for a semilinear elliptic system without variational structure and their applications, Math. Ann. 323 (2002), 713-735.

Yajing Zhang

School of Mathematical Sciences

Shanxi University

Taiyuan 030006

P.R. China

and

Institute of Mathematics

AMSS

Chinese Academy of Sciences

Beijing 100080

P.R. China

e-mail: zhangyj@sxu.edu.cn 
Jianghao Hao

School of Mathematical Sciences

Shanxi University

Taiyuan 030006

P.R. China

e-mail: hjhao@sxu.edu.cn

Received: 20 September 2007.

Revised: 24 December 2007.

Accepted: 27 January 2008. 